\title{
Piezoelectric Nanogenerator Based on Lead-Free Flexible PVDF-Barium Titanate Composite Films for Driving Low Power Electronics
}

\author{
Manisha Sahu ${ }^{1}$, Sugato Hajra ${ }^{1}$, Kyungtaek Lee ${ }^{1} \mathbb{D}$, PL Deepti ${ }^{2}$, Krystian Mistewicz ${ }^{3}$ and Hoe Joon Kim ${ }^{1, *(D)}$ \\ 1 Department of Robotics Engineering, Daegu Gyeongbuk Institute of Science \& Technology (DGIST), \\ Daegu 42988, Korea; manishasahu@dgist.ac.kr (M.S.); sugatofl@outlook.com (S.H.); 1kt4215@dgist.ac.kr (K.L.) \\ 2 Department of Physics, Veer Surendra Sai University of Technology, Odisha 768018, India; \\ pldeepti@rediffmail.com \\ 3 Institute of Physics-Centre for Science and Education, Silesian University of Technology, \\ 44100 Katowice, Poland; krystian.mistewicz@polsl.pl \\ * Correspondence: joonkim@dgist.ac.kr
}

Citation: Sahu, M.; Hajra, S.; Lee, K.; Deepti, P.; Mistewicz, K.; Kim, H.J. Piezoelectric Nanogenerator Based on Lead-Free Flexible PVDF-Barium Titanate Composite Films for Driving Low Power Electronics. Crystals 2021, 11, 85. https://doi.org/10.3390/ cryst11020085

Received: 30 December 2020

Accepted: 20 January 2021

Published: 21 January 2021

Publisher's Note: MDPI stays neutral with regard to jurisdictional claims in published maps and institutional affiliations.

Copyright: (c) 2021 by the authors. Licensee MDPI, Basel, Switzerland. This article is an open access article distributed under the terms and conditions of the Creative Commons Attribution (CC BY) license (https:// creativecommons.org/licenses/by/ $4.0 /)$.

\begin{abstract}
Self-powered sensor development is moving towards miniaturization and requires a suitable power source for its operation. The piezoelectric nanogenerator (PENG) is a potential candidate to act as a partial solution to suppress the burgeoning energy demand. The present work is focused on the development of the PENG based on flexible polymer-ceramic composite films. The $X$-ray spectra suggest that the BTO particles have tetragonal symmetry and the PVDF-BTO composite films (CF) have a mixed phase. The dielectric constant increases with the introduction of the particles in the PVDF polymer and the loss of the CF is much less for all compositions. The BTO particles have a wide structural diversity and are lead-free, which can be further employed to make a CF. An attempt was made to design a robust, scalable, and cost-effective piezoelectric nanogenerator based on the PVDF-BTO CFs. The solvent casting route was a facile approach, with respect to spin coating, electrospinning, or sonication routes. The introduction of the BTO particles into PVDF enhanced the dielectric constant and polarization of the composite film. Furthermore, the single-layered device output could be increased by strategies such as internal polarization amplification, which was confirmed with the help of the polarization-electric field loop of the PVDF-BTO composite film. The piezoelectric nanogenerator with $10 \mathrm{wt} \%$ BTO-PVDF CF gives a high electrical output of voltage $7.2 \mathrm{~V}$, current $38 \mathrm{nA}$, and power density of $0.8 \mu \mathrm{W} / \mathrm{cm}^{2}$ at $100 \mathrm{M} \Omega$. Finally, the energy harvesting using the fabricated PENG is done by various actives like coin dropping, under air blowing, and finger tapping. Finally, low-power electronics such as calculator is successfully powered by charging a $10 \mu \mathrm{F}$ capacitor using the PENG device.
\end{abstract}

Keywords: dielectric; lead-free; piezoelectric nanogenerator; energy harvesting

\section{Introduction}

In the present era, the development of a sustainable and flexible energy harvester that harvests energy from listed mechanical sources to power electronics and nanosystems is attaining huge attention [1,2]. Nanogenerators provide a pathway towards sustainable power sources due to their superior characteristics of easy, less cost fabrication, mass production, improved energy conversion efficiency [3,4]. Currently, the quest is to develop flexible nanogenerators working on principles of piezoelectricity [5] and triboelectricity [6]. Nanogenerators based on the piezoelectric principle have gained immense attention due to many advantages such as wide piezoelectric materials selection, easy fabrication, and quick response $[7,8]$. The various structure and shapes of the flexible piezoelectric nanogenerator can be achieved by using several polymers like PVDF, PVDF-copolymer [9], PDMS [10], Nylon [11], and polyamide [12]. Among these, PVDF and its copolymers are chosen for 
several studies owing to its good electromechanical coupling, chemical stability, and high piezoelectric coefficient $[13,14]$.

The PVDF has five crystalline polymorphs such as $\alpha, \beta, \gamma, \delta$, and $\varepsilon$ phases $[15,16]$. The presentation of the phases in the PVDF solely depends on the processing methodology [17]. The $\beta$ phase bears the highest electroactive properties [18]. There are several strategies followed to achieve the electroactive $\beta$ phase, such as applying stretching mechanically with heating, applying high voltage, and doping of piezoelectric materials or metallic nanoparticles $[19,20]$. Mokhtari et al. explored the textile architecture and achieved an energy conversion efficiency of $22.5 \%$ for the coiled hybrid piezo fiber energy generator [21]. Mokhtari and co-workers tested pizeofibers that were capable of producing a maximum voltage output of $4 \mathrm{~V}$ and a power density of $87 \mu \mathrm{W} \cdot \mathrm{cm}^{-3}$, which were 45 times higher than earlier reported for piezoelectric textiles [22]. Pereria et al. fabricated the BTO-PVDF-TRFE composites and achieved a power output of $0.28 \mu \mathrm{W}$ [23]. Zhao et al. suggested that the

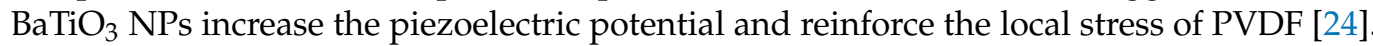
Ponnamma et al. fabricated hybrid composite ( $3 \mathrm{wt} \% \mathrm{BaTiO}_{3}, \mathrm{PVDF}-\mathrm{HFP}, 1 \mathrm{wt} \% \mathrm{~h}-\mathrm{BN}$ ) generated a piezoelectric output voltage $(2.4 \mathrm{~V})$ with high dielectric constant 45 and low dielectric loss 7.8 [25]. PVDF-ZnO-BTO composite film (CF) was fabricated by Sabry et al. that was capable of generating $11 \mathrm{~V}$ at $1.328 \mathrm{~N}$ force [26]. The authors added a comparison table of the various PVDF-ceramic composites, their preparation approach, and electrical output response in Table S1.

Perovskite ferroelectric oxides are one of the widely investigated materials with a general chemical formula of $\mathrm{ABO}_{3}$ [27]. BTO ceramics are categorized under the oxygen octahedron group. $\mathrm{BaTiO}_{3}$ undergoes the ferroelectric-paraelectric transition $\left(\mathrm{T}_{\mathrm{c}}\right)$ about $120{ }^{\circ} \mathrm{C}$ [28]. It exhibits a cubic structure (space group Pm3m) above the $\mathrm{T}_{\mathrm{c}}$, and below this temperature, it bears a tetragonal structure (space group $\mathrm{P} 4 \mathrm{~mm}$ ). In the cubic symmetry, the $\mathrm{Ti}^{4+}$ ion present in the oxygen octahedra center causing the non-ferroelectric behavior of BTO. During the tetragonal symmetry, BTO bears ferroelectric properties as $\mathrm{Ti}^{4+}$ ion shifts from the center towards one of the two oxygen ions along the axis. BTO bears more structural changes such as the temperature declines from $120^{\circ} \mathrm{C}$ to below, it has orthorhombic crystal symmetry (space group $A m m 2$ ) at $5{ }^{\circ} \mathrm{C}$, at $-90{ }^{\circ} \mathrm{C}$ it transforms to rhombohedral crystal symmetry, and at $1460{ }^{\circ} \mathrm{C}$ it transforms to hexagonal crystal symmetry. BTO is a lead-free ceramic with reported piezoelectric coefficient values ranging from 350 to $460 \mathrm{pC} / \mathrm{N}$ by several synthesis routes, and it can act as an alternative towards the toxic lead-based compositions $[29,30]$. The polymer ceramic composites have several merits such as mechanical flexibility and rather simple process to control the film thicknesses and sizes [31,32]. The limitations of the high brittle nature of the BTO ceramics can be resolved by integrating in particle forms into the PVDF, further improving the electroactive phase of PVDF.

The present work focuses on the fabrication of the thin polymer-ceramic CFs via solvent casting route. Mixed phases of the PVDF and BTO are seen from the XRD spectra confirming the crystallinity and the distribution of the particles within the matrix is depicted from the SEM micrographs. The improved dielectric constant and low loss of the PVDF-BTO CF is also confirmed. The flexible piezoelectric nanogenerator is designed and experiment with in terms of the different $\mathrm{wt} \%$, and with the optimized PENG device, other experiments and several testing of charging capacitors and the powering calculator is carried out. The solvent casting route is a cheap fabrication technique that has been adapted for this work as a facile approach, as compared to complicated spin coating, electrospinning, or sonication routes. The introduction of the BTO particles into PVDF leads to the improvement of the dielectric constant and polarization. The Polarization-electric field loop confirms that the BTO particles could enhance the internal polarization of the PVDF. Furthermore, the single-layered device output can be increased by strategies such as internal polarization amplification. This work paves the way to fabricate new piezoelectric material for energy harvesting. 


\section{Processing and Experimental Techniques}

\subsection{Synthesis of $\mathrm{BaTiO}_{3}$ Particles}

The BTO particles were synthesized by mixing the various precursors and heat treatment at high temperature. The high purity barium carbonate and titanium oxide were procured from Loba Chemie, Mumbai, India. The stoichiometric proportion of the powders were carefully measured using a digital weighing balance. The powders were transferred to an agate mortar and mixed for $2 \mathrm{~h}$ in a methanol medium. The homogeneously mixed powders were put into an alumina boat and calcined inside a box furnace at $1200{ }^{\circ} \mathrm{C}$ for $4 \mathrm{~h}$. Further, the lump was ground into fine particles utilizing an agate mortar and pestle.

\subsection{Synthesis of PVDF-BaTiO ${ }_{3}$ Composite Film and PENG Device}

The composite film (CF) of the PVDF-BTO was prepared by a cost-effective solvent casting method. For this, a solvent of N-Methyl-2-Pyrrolidone (NMP) was procured from Sisco Research Ltd., Mumbai, India. The PVDF pellets were procured from Sigma Aldrich, St. Louis, MO, USA. The PVDF pellets were measured in proper ratio and put into the NMP solvent at $60^{\circ} \mathrm{C}$ upon a magnetic stirrer with a magnetic bead inside it. The clear transparent solution was obtained after $1 \mathrm{~h}$, and the various $\mathrm{wt} \%$ of the BTO particles were put into the solution. Dispersions with different concentrations of BTO $(1 \mathrm{wt} \%, 5 \mathrm{wt} \%$, $7 \mathrm{wt} \%, 10 \mathrm{wt} \%$ ) were prepared. Further, it was allowed to mix for $1 \mathrm{~h}$ at room temperature until a homogenous mixture was achieved. Then the PVDF-BTO solution was poured onto a glass Petri plate and left inside an electric oven at $70{ }^{\circ} \mathrm{C}$ for two hours. After curing, a free-standing flexible PVDF-BTO CF was obtained.

For the PENG device fabrication, the film was cut into dimensions of $1.5 \mathrm{~cm} \times 1.5 \mathrm{~cm}$, and the gold coating was done to act as an electrode. The electrode area was kept as $1 \mathrm{~cm} \times 1 \mathrm{~cm}$. Further, to measure the system's energy output, thin copper tape was attached to the opposite side of the device. It was then transferred to antistatic tape to neglect any of the electrostatic charges present in the environment. Then the entire device was encapsulated using a PDMS layer. The PENG devices were poled at $1 \mathrm{kV}$ for $30 \mathrm{~min}$ at RT to align dipoles.

\subsection{Experimental Techniques}

The structural analysis of the BTO particles and PVDF-BTO CFs were performed using a powder X-ray diffraction (PANalytical Empyrean, Almelo, The Netherlands, $\mathrm{Cu}-\mathrm{K} \alpha$ radiation) under a scanning step size of $3^{\circ} / \mathrm{min}$. The surface morphology was taken using a Hitachi S-4800 scanning electron microscope fitted with an EDS detector. The CF was cut into small pieces, and the electrode was done with gold sputtering onto opposite sides for electrical characterization at room temperature. The dielectric measurement was taken using an N4L PSM LCR meter. The periodic contact-separation of the device was performed by a linear motor (LinMot Inc., Lake Geneva, WI, USA). An electrometer 6514 (Keithley, Solon, OH, USA) was used to measured voltage and current from the piezoelectric device.

\section{Results and Discussion}

The systematic synthesis route is the possible way to properly obtain a high crystalline material and with superior properties. In Figure 1a, the mixing of the high purity barium carbonate and titanium oxide is mixed using a mortar and pestle in the presence of the methanol for $4 \mathrm{~h}$ and then transferred into an alumina crucible. The mixture is calcined at $1200{ }^{\circ} \mathrm{C}$ for $4 \mathrm{~h}$. The morphological properties, such as particle size and shape of prepared BTO particles were analyzed from the captured SEM image, as shown in Figure 1a. The SEM image reveals the polyhedron shape of particles with a very smooth surface and edges. The particle size histogram with a Gaussian peak fitted curve is shown in Figure S1a. It depicts the presence of particles having a size distribution in the range of 200 to $1300 \mathrm{~nm}$. The average particle size was about $460 \mathrm{~nm}$, which was calibrated from the Gaussian peak fitting of the particle size distribution histogram. Figure $1 \mathrm{~b}$ shows the formation of the PVDF-BTO CF. In this, the PVDF pellets are dissolved in the NMP solvent and heated at 
$60{ }^{\circ} \mathrm{C}$ for $1 \mathrm{~h}$ using a magnetic stirring. Then the BTO particles are added to the transparent solution and the temperature is reduced to room temperature with continuous mixing giving rise to a PVDF-BTO homogenous solution. It is then poured onto a glass Petri dish and left for curing. The contact angle measurement of the PVDF-BTO films shows an angle greater than 100 degrees, which suggests the higher hydrophobic nature of the films. Figure 1c shows the XRD pattern of the BTO particles at room temperature. The peaks are well-matched with reported JCPDS data: 01-075-0583. The POWD MULT software was used to derive the lattice parameters following a least-square refinement rule. The values of a: $3.988(\AA ీ)$; c: $3.979(\AA ీ)$; volume: $64.2\left(\AA^{3}\right)$. The XRD peaks of the polymer ceramic CFs showed the presence of both PVDF and BTO phases. Various symbols were used to represent the PVDF phase and the perovskite phase of BTO. It was depicted that a higher concentration of $10 \mathrm{wt} \%$ into the PVDF leads to an increase in the BTO phase and a reduction in the PVDF phase intensity. It can be also noticed that the phase of the BTO has not been altered as it gets dissolved in PVDF, which can be confirmed as there is no peak shifting occurred in the polymer-ceramic CF.

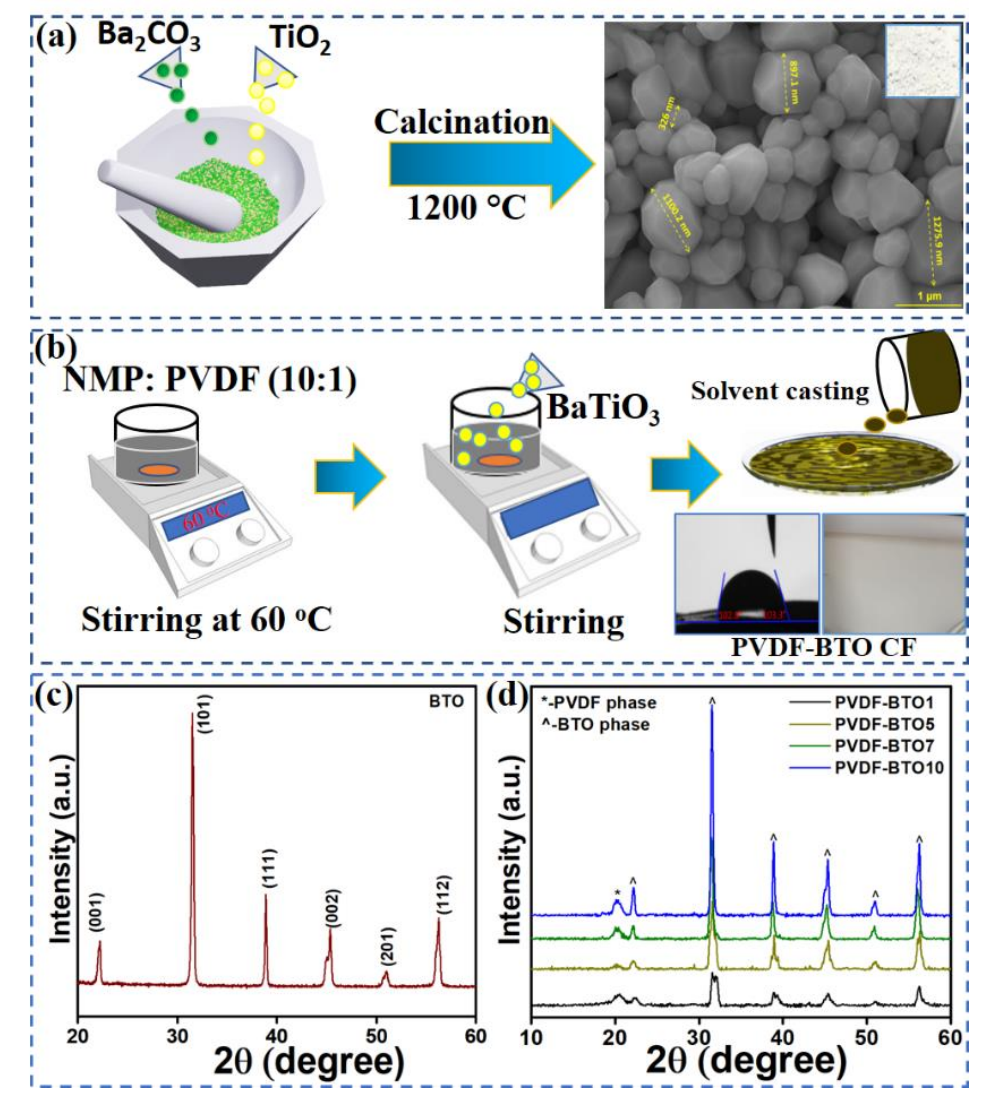

Figure 1. Synthesis of particle and piezoelectric nanogenerator; (a) $\mathrm{BaTiO}_{3}$ particles by solid-state reaction method and surface morphology of the polycrystalline particles; (b) the solvent casting route and systematic steps for formation of the PVDF-BTO composite film, the contact angle of the composite film showing hydrophobic nature of their surfaces; (c) XRD pattern of the $\mathrm{BaTiO}_{3}$ particles

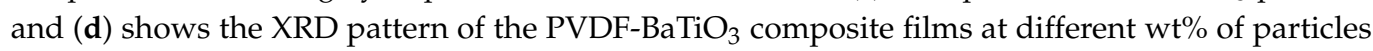
$(1,5,7,10 \mathrm{wt} \%)$ into the PVDF matrix.

Figure 2a shows the surface morphology of the CF. It shows that the BTO concentration increases in the PVDF and there is no clustering of the particles. The $10 \mathrm{wt} \%$ BTO-PVDF CF show more compactness as compared to other CF. The EDS spectra of the $10 \mathrm{wt} \%$ BTO-PVDF shows that all the elements are present without any trace of impurity. Figure $2 b$ shows the frequency-dependent dielectric constant at room temperature. It shows that the dielectric contact rises as the BTO particles in the PVDF increases. The pure PVDF shows 
23 dielectric constant values at $1 \mathrm{kHz}$ while the $10 \mathrm{wt} \%$ BTO-PVDF CF shows 47 at $1 \mathrm{kHz}$, suggesting that there is a rise in connectivity between the particles as the BTO content rises to improve the dipole-dipole interaction [33]. The reason behind the increase in the dielectric constant in low frequency is mainly due to the effect of various polarizations, and with a rise in frequency, the polarization activity fades, leading to constant or reduced dielectric constant magnitude. Figure $2 c$ shows the dielectric loss versus frequency at room temperature for various CF. The interfacial polarization could lead to a rise in the loss factor in the low-frequency region. The heterogeneity in the $\mathrm{CF}$, with a rise in the BTO content in the PVDF, is also responsible for increasing the loss factor as the BTO. Another possible cause may be from the air voids ionization, which creates plasma as an electric field is applied to the CF. Figure $2 \mathrm{~d}$ shows the digital image of the fabricated piezoelectric device, which is flexible and bendable. The ferroelectric polarization of the pure PVDF and PVDF-BTO (10 wt \%) is compared in Figure S1b. The remnant polarization of the PVDF is $0.52 \mu \mathrm{C} / \mathrm{cm}^{2}$, whereas for the PVDF-BTO CF it is $0.66 \mu \mathrm{C} / \mathrm{cm}^{2}$. It demonstrates that as the BTO content increases in the PVDF, the dielectric constant and ferroelectric polarization are both improved in the material. It is also noted that the relation below links the dielectric property to the piezoelectric voltage co-efficient: $g_{i j}=d_{i j} /\left(\varepsilon_{0} \varepsilon_{r}\right)$. Therefore, as the dielectric constant increases, the piezoelectric output response should also rise.
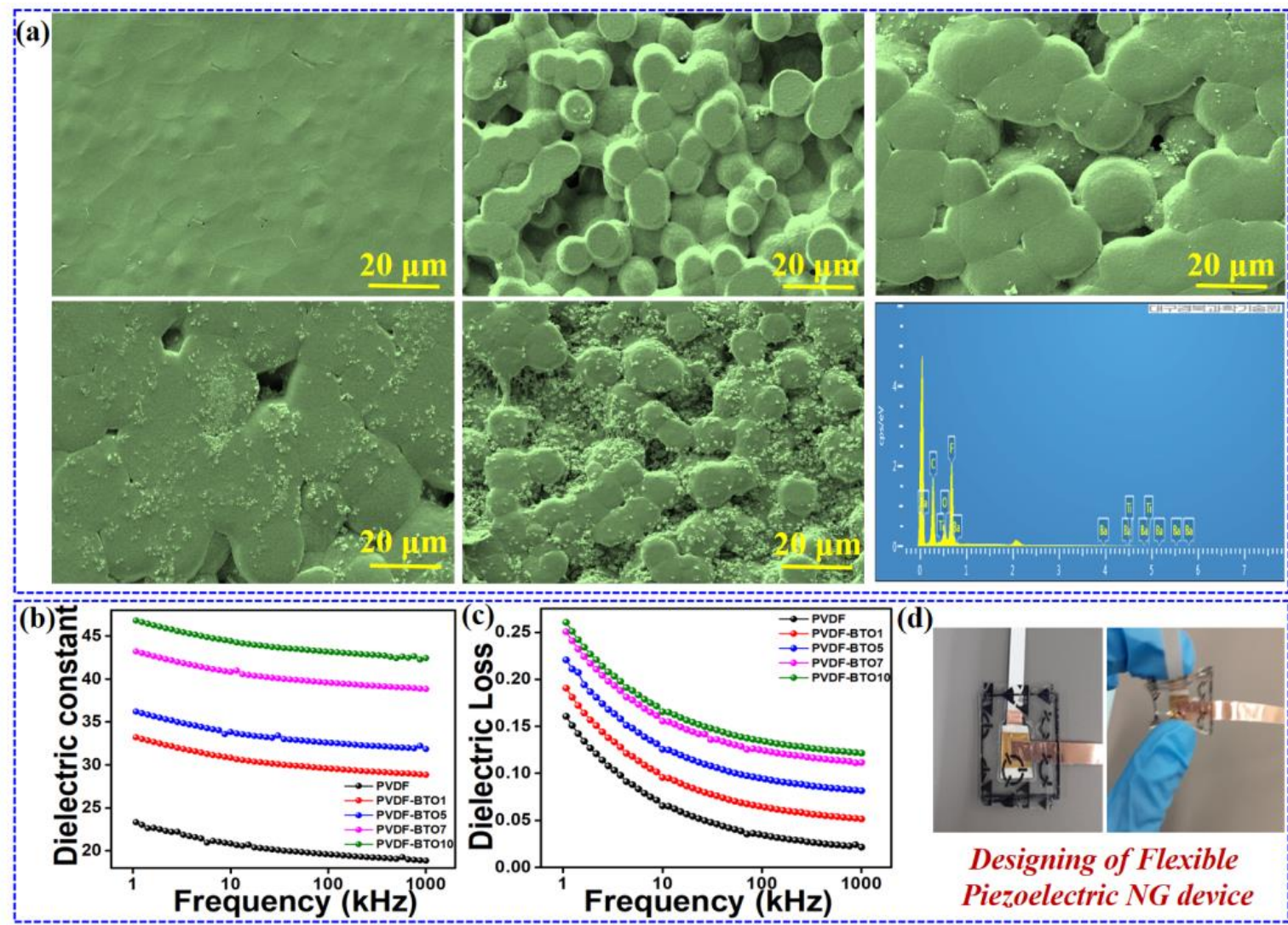

Figure 2. Properties of the PVDF-BTO composite films; (a) the surface morphology of pure PVDF and PVDF-BTO composite films $(1,5,7,10 \mathrm{wt} \%)$; the EDS spectra of the PVDF-BTO of 10wt\% BTO into PVDF is examined, and all the elements match well with its chemical composition; $(\mathbf{b}, \mathbf{c})$ dielectric constant and dielectric loss of the pure PVDF and composite films; (d) the image of the flexible PNG10 piezoelectric nanogenerator device.

Figure 3a shows the working mechanism of the piezoelectric nanogenerator device. In general, if a mechanical force is applied to a PENG device, there is the generation of positive and negative piezoelectric potential in the materials leading to an electrical output at both ends of electrodes. It is seen as the pressure is acted upon by the piezoelectric nanogenerator, the electrons flow from one end to the other, leading to the flow of current 
through an external load. As the force is removed, the stored electrons move through the external circuit in a reverse manner creating a negative voltage. This repeated process leads to the generation of the output of PENG. Figure $3 \mathrm{~b}$ shows the representation of the dipoles; as we apply a voltage of $1 \mathrm{kV}$ across the piezoelectric $\mathrm{CF}$ the dipoles are aligned, which is required to achieve higher piezoelectric output. Figure $3 \mathrm{c}$ shows the layer by layer arrangement of the PENG device; it has several layers sandwiched into one structure. The antistatic tape is used to remove any stray charges in the air atmosphere while the PDMS encapsulation act as a packing layer. Figure $3 \mathrm{~d}$, e shows the voltage and current output of the various PENG devices. The $1 \mathrm{wt} \%$ BTO in PVDF (PNG1), $5 \mathrm{wt} \%$ BTO in PVDF (PNG5), $7 \mathrm{wt} \%$ BTO in PVDF (PNG7), and $10 \mathrm{wt} \%$ BTO in PVDF (PNG10) are the abbreviated names of the fabricated PENG devices. It is seen that the PNG20 shows a higher output of voltage $7.2 \mathrm{~V} /$ current $38 \mathrm{nA}$. Figure $3 \mathrm{f}$ presents the switching polarity test confirming that the generated outputs are due solely to the piezoelectric effect. It is to note that no change in phase in the peak pattern, indicative of a true piezoelectric response.

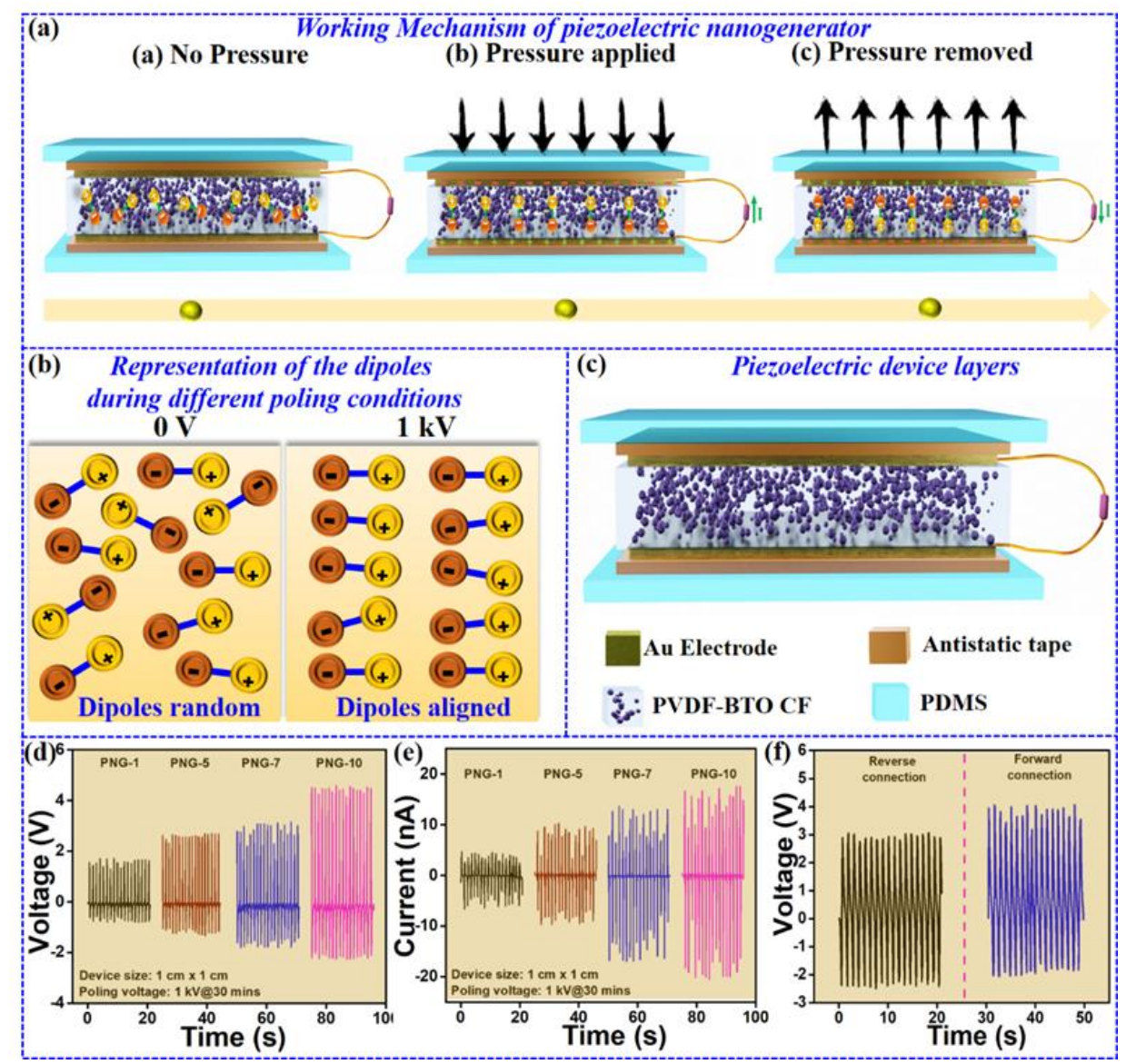

Figure 3. Experimental for the piezoelectric nanogenerator. (a) Working mechanism of the fabricated piezoelectric generator, (b) representation of the poling and dipole arrangement on applying different voltage, (c) layer by layer schematic of the piezoelectric nanogenerator device, (d,e) voltage and current of the piezoelectric nanogenerator of various composition, (f) the reverse and forward voltage/switching polarity test of the PNG10 device.

Figure $4 \mathrm{a}, \mathrm{b}$ shows the current and voltage of the PNG10 device at various accelerations/frequencies. It is seen that at higher acceleration/frequencies, the output of the device is not uniform as the device may be depolarized with higher acceleration. Figure $4 \mathrm{c}$ shows the loading matching analysis of the PNG10 device. The power density $\left(P=V^{2} / R\right)$ of the PNG10 device under optimized conditions is determined by varying the load matching $R$ from $k \Omega$ to $G \Omega$ and measuring the corresponding voltage. It was observed that a 
power density of $0.8 \mu \mathrm{W} / \mathrm{cm}^{2}$ at $100 \mathrm{M} \Omega$ was achieved by the PNG10 device. Figure $4 \mathrm{~d}$ shows the charging of the various capacitor with different capacitance. Figure 4e shows the charging and discharging curve of the $4.7 \mu \mathrm{F}$ capacitor several times, which shed light upon the fact that the output generated by the PNG10 device is stable for a long time. Figure $4 \mathrm{f}$ shows the stored charge in each capacitor that was calculated using the formula $\mathrm{Q}=\mathrm{CV}$.
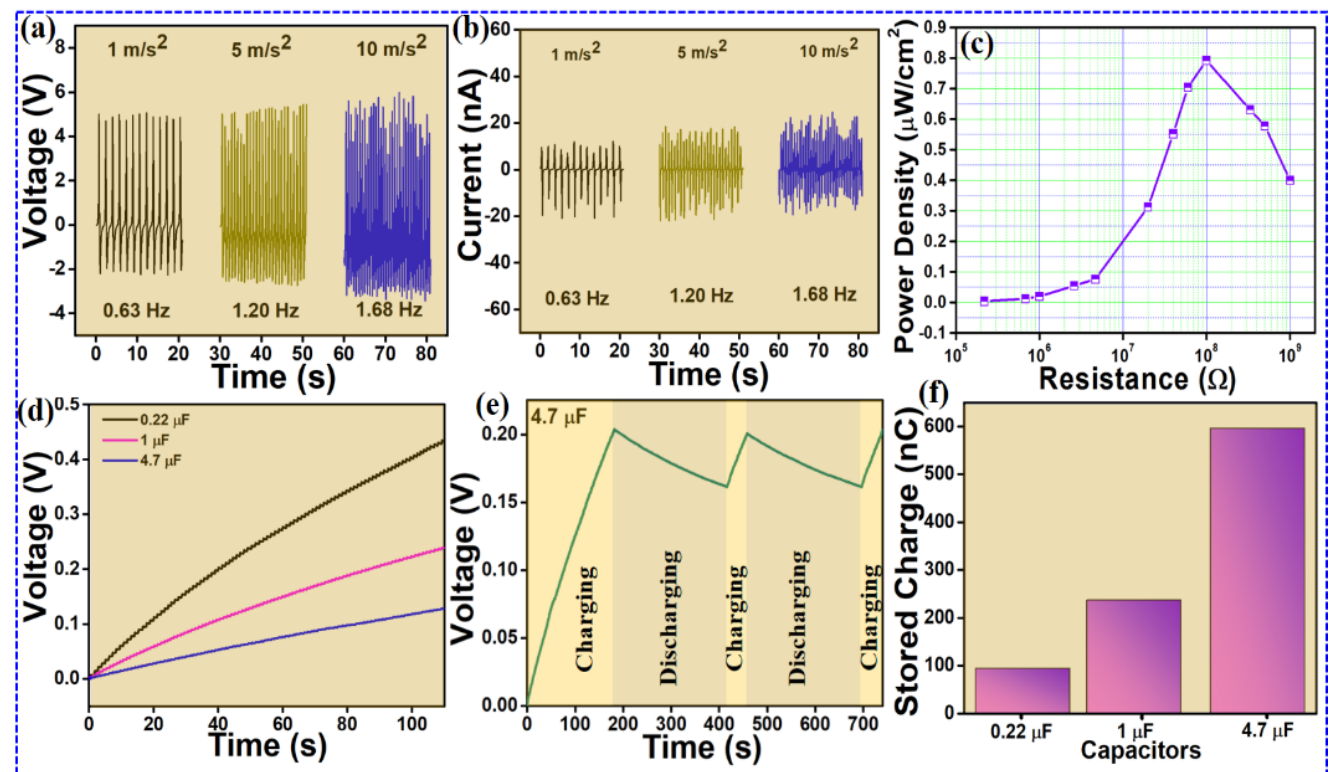

Figure 4. Electrical response of the piezoelectric nanogenerator. $(\mathbf{a}, \mathbf{b})$ Voltage and current measurement of the PNG10 device at various accelerations/frequencies, (c) the instantaneous output power density as a function of different external load resistance, (d) charging curve of two capacitors with different capacitances, (e) charging and discharging curve of the $4.7 \mu \mathrm{F}$ capacitors showing stable output from the device, (f) the amount of stored charge in the different capacitors.

The real-time energy harvesting from the PNG10 was carried out using several activities. Figure 5 shows the output voltage when a coin was dropped upon the device PNG10 it could sense the impact from the dropping of the coin. In Figure 5b, the PNG10 device was connected to the fan, and when the fan was turned on at its highest speed, the device could produce $6.9 \mathrm{~V}$. Figure $5 \mathrm{c}$, $\mathrm{d}$ demonstrates the biomechanical energy harvesting from the PNG10 device was tested by simple finger tapping and the voltage of $1.4 \mathrm{~V}$ and current of $24 \mathrm{nA}$. To demonstrate, the powering of the calculator in Figure 5e,f, two PNG10 devices were connected in parallel to increase the current produced from the device, and this was utilized directly to charge a $10 \mu \mathrm{F}$ capacitor. The AC signal produced by the two PNG10 devices is converted to DC via a bridge rectifier circuit. When the switch was ON, the calculator began to work and the capacitor was discharged. Figure $5 \mathrm{f}$ shows the digital image of the demonstration of the powering of the calculator. Hence the fabrication of the PVDF-BTO CFs based flexible nanogenerator paves the way towards a sustainable power source and a promising entrant for designing self-power applications. Figure S2a shows the result of the manual bending test: as the device is flexible, it could easily bend and produce an electrical output. Figure S2b,c show the electrical response of the device while performing an exercise like a basic squat exercise. The device generated an electrical voltage of $6 \mathrm{~V}$ and a current of $19 \mathrm{nA}$, demonstrating that the presented PENG device can be used for counting the number of times the exercise was performed. 

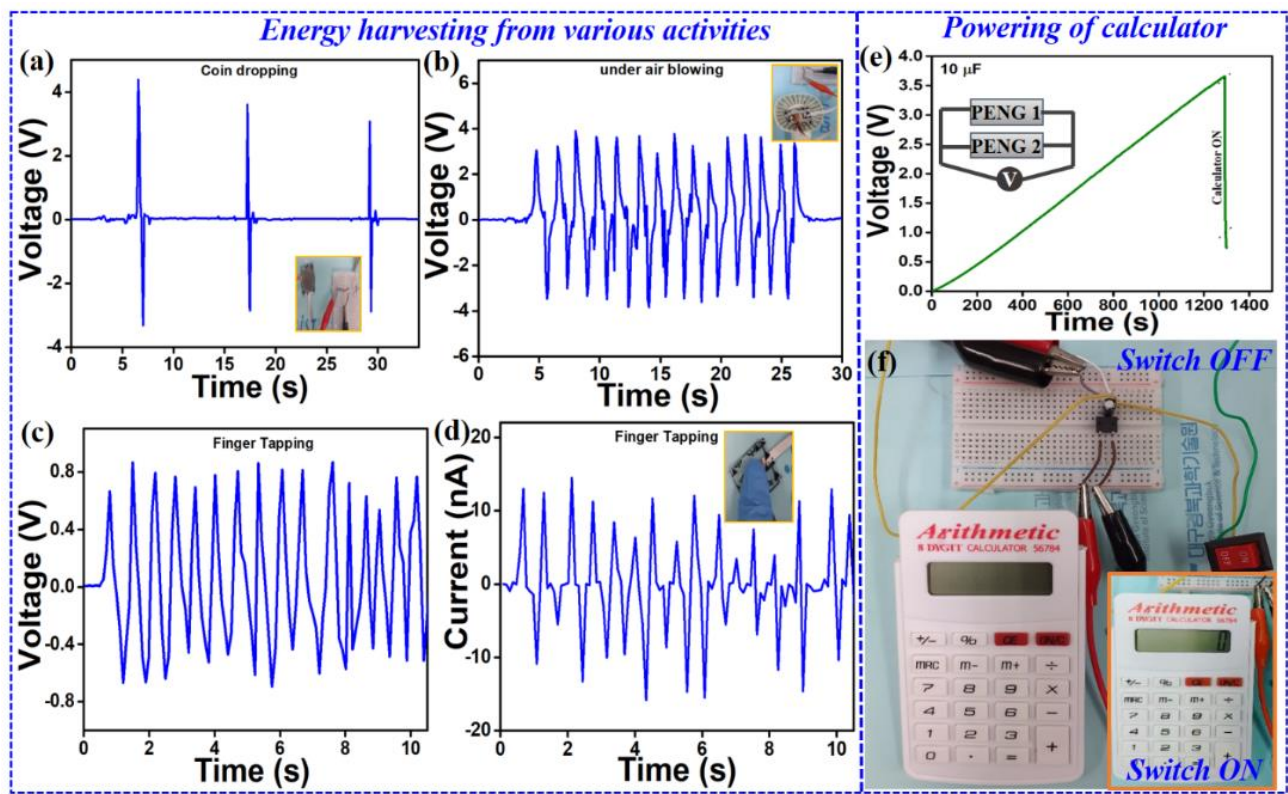

Figure 5. Applications of harvesting energy and powering electronics. (a) Electrical response of PNG10 when a coin drop upon it, (b) Electrical response of the PNG-10 device when connected to fan; (c,d) Voltage and current response of PNG10 device upon finger tapping, (e) connected two PNG10 devices in parallel and charge a $10 \mu \mathrm{F}$ capacitor, and (f) Power supply to low power rating devices like calculator (switch OFF and switch ON condition).

\section{Conclusions}

The PVDF-BTO composite films are highly flexible and synthesized via cost-effective processing known as the solvent casting route. The XRD pattern confirms the crystallinity features, and the surface morphology suggests that the particles are uniformly distributed upon the PVDF. The BTO particles crystallize with tetragonal symmetry. The dielectric constant increases with the doping of the BTO particles into the PVDF, showing that there are dipole-dipole interactions. Individual PENG devices are tested after the polling condition. The PNG10 device delivers an output of voltage $7.2 \mathrm{~V}$ and $38 \mathrm{nA}$. An instantaneous power density of $0.8 \mu \mathrm{W} / \mathrm{cm}^{2}$ at a matching resistance of $100 \mathrm{M} \Omega$ is achieved from the PNG10 device. The stable output of the device can be seen from the continuous charging and discharging curve of the commercial capacitor. The PENG device can power up the calculator via a bridge rectifier, switch, and charging capacitor of $10 \mu \mathrm{F}$. The proposed device fabrication is adaptable and straightforward to harness energy from different vibrations or motions in the environment. It is many benefits like eco-friendly, cost-effective, and easily scalable methods to meet the power requirements of micro/nano electronic devices in the future.

Supplementary Materials: The following are available online at https:/ / www.mdpi.com/2073-435 2/11/2/85/s1, Figure S1: (a) A particle size distribution histogram determined from the SEM images of BTO particles; (b) P-E hysteresis loop of PVDF and PVDF-BTO (10 wt \%), Figure S2: (a) Electrical response of the PENG device upon the hand bending; $(b, c)$ voltage and current of the PENG device upon the repetitive Squat exercise Table S1: Comparison of the piezoelectric nanogenerator based on polymer ceramic composites; device output and composite film preparation.

Author Contributions: Conceptualization, S.H.; methodology, M.S.; formal analysis, K.L. and M.S.; data curation, P.D.; writing — original draft preparation, M.S. and S.H.; writing — review and editing, K.M., S.H. and H.J.K.; supervision, H.J.K.; funding acquisition, H.J.K. All authors have read and agreed to the published version of the manuscript. 
Funding: This study is funded by the Basic Science Research Program through the National Research Foundation of Korea (NRF) and funded by the Ministry of Science and ICT of Korea (2018R1C1B6008041) and the DGIST R\&D Program of the Ministry of Science and ICT of Korea (19-RT-01).

Institutional Review Board Statement: Not applicable.

Informed Consent Statement: Not applicable.

Data Availability Statement: Not applicable.

Conflicts of Interest: The authors declare no conflict of interest.

\section{References}

1. Chen, J.; Wang, Z.L. Reviving vibration energy harvesting and self-powered sensing by a triboelectric nanogenerator. Joule 2017, 1, 480-521. [CrossRef]

2. Sahu, M.; Vivekananthan, V.; Hajra, S.; Sahu, M.; Vivekananthan, V.; Hajra, S.; Abisegapriyan, K.S.; Raj, N.P.M.J.; Kim, S.J. Synergetic enhancement of energy harvesting performance in triboelectric nanogenerator using ferroelectric polarization for self-powered IR signaling and body activity monitoring. J. Mater. Chem. A 2020, 8, 22257-22268. [CrossRef]

3. Wang, Y.; Yang, Y.; Wang, Z.L. Triboelectric nanogenerators as flexible power sources. NPJ Flex. Electron. 2017, 1, 1-10. [CrossRef]

4. Kang, X.; Pan, C.; Chen, Y.; Pu, X. Boosting performances of triboelectric nanogenerators by optimizing dielectric properties and thickness of electrification layer. RSC Adv. 2020, 10, 17752-17759. [CrossRef]

5. Le, A.T.; Ahmadipour, M.; Pung, S.Y. A review on ZnO-based piezoelectric nanogenerators: Synthesis, characterization techniques, performance enhancement and applications. J. Alloys Compd. 2020, 844, 156172. [CrossRef]

6. Zhou, L.; Liu, D.; Wang, J.; Wang, Z.L. Triboelectric nanogenerators: Fundamental physics and potential applications. Friction 2020, 8, 481-506. [CrossRef]

7. Szewczyk, P.K.; Gradys, A.; Kim, S.K.; Persano, L.; Marzec, M.; Kryshtal, A.; Busolo, T.; Toncelli, A.; Pisignano, D.; Bernasik, A.; et al. Enhanced piezoelectricity of electrospun polyvinylidene fluoride fibers for energy harvesting. ACS Appl. Mater. Interfaces 2020, 12, 13575-13583. [CrossRef]

8. Dudem, B.; Bharat, L.K.; Patnam, H.; Mule, A.R.; Yu, J.S. Enhancing the output performance of hybrid nanogenerators based on Al-doped BaTiO3 composite films: A self-powered utility system for portable electronics. J. Mater. Chem. A 2018, 6, 16101-16110. [CrossRef]

9. Gaur, A.; Tiwari, S.; Kumar, C.; Maiti, P. Bio-waste orange peel and polymer hybrid for efficient energy harvesting. Energy Rep. 2020, 6, 490-496. [CrossRef]

10. Vivekananthan, V.; Chandrasekhar, A.; Alluri, N.R.; Purusothaman, Y.; Kim, W.J.; Kang, C.-N.; Kim, S.-J. A flexible piezoelectric composite nanogenerator based on doping enhanced lead-free nanoparticles. Mater. Lett. 2019, 249, 73-76. [CrossRef]

11. Newman, B.A.; Chen, P.; Pae, K.D.; Scheinbeim, J.I. Piezoelectricity in nylon 11. J. Appl. Phys. 1980, 51, 5161-5164. [CrossRef]

12. Soin, N.; Zhao, P.; Prashanthi, K.; Chen, J.; Ding, P.; Zhou, E.; Luo, J. High performance triboelectric nanogenerators based on phase-inversion piezoelectric membranes of poly (vinylidene fluoride)-zinc stannate (PVDF-ZnSnO 3 ) and polyamide-6 (PA6). Nano Energy 2016, 30, 470-480. [CrossRef]

13. Park, S.; Kim, Y.; Jung, H.; Park, J.Y.; Lee, N.; Seo, Y. Energy harvesting efficiency of piezoelectric polymer film with graphene and metal electrodes. Sci. Rep. 2017, 7, 17290. [CrossRef] [PubMed]

14. Levi, N.; Czerw, R.; Xing, S.; Iyer, P.; Carroll, D.L. Properties of polyvinylidene difluoride-Carbon nanotube blends. Nano Lett. 2004, 4, 1267-1271. [CrossRef]

15. Cai, X.; Lei, T.; Sun, D.; Lin, L. A critical analysis of the $\alpha, \beta$ and $\gamma$ phases in poly (vinylidene fluoride) using FTIR. RSC Adv. 2017, 7, 15382-15389. [CrossRef]

16. Sukumaran, S.; Chatbouri, S.; Rouxel, D.; Tisserand, E.; Thiebaud, F.; Ben Zineb, T. Recent advances in flexible PVDF based piezoelectric polymer devices for energy harvesting applications. J. Intell. Mater. Syst. Struct. 2020. [CrossRef]

17. Harstad, S.; D'Souza, N.; Soin, N.; El-Gendy, A.A.; Gupta, S.; Pecharsky, V.; Shah, T.; Siores, E.; Hadimani, R.L. Enhancement of $\beta$-phase in PVDF films embedded with ferromagnetic Gd5Si4 nanoparticles for piezoelectric energy harvesting. AIP Adv. 2017, 7, 056411. [CrossRef]

18. Ghosh, S.K.; Mandal, D. High-performance bio-piezoelectric nanogenerator made with fish scale. Appl. Phys. Lett. 2016, 109, 103701. [CrossRef]

19. Bodkhe, S.; Rajesh, P.S.M.; Kamle, S.; Verma, V. Beta-phase enhancement in polyvinylidene fluoride through filler addition: Comparing cellulose with carbon nanotubes and clay. J. Polym. Res. 2014, 21, 1-11. [CrossRef]

20. Huang, Z.-J.; Jiang, J.; Xue, G.; Zhou, D. $\beta$-Phase crystallization of poly(vinylidene fluoride) in poly(vinylidene fluoride)/poly(ethyl methacrylate) blends. Chin. J. Polym. Sci. 2018, 37, 94-100. [CrossRef]

21. Mokhtari, F.; Spinks, G.M.; Sayyar, S.; Cheng, Z.; Ruhparwar, A.; Foroughi, J. Highly stretchable self-powered wearable electrical energy generator and sensors. Adv. Mater. Technol. 2020, 2000841. [CrossRef]

22. Mokhtari, F.; Spinks, G.M.; Fay, C.; Cheng, Z.; Raad, R.; Xi, J.; Foroughi, J. Wearable electronic textiles from nanostructured piezoelectric fibers. Adv. Mater. Technol. 2020, 5, 1900900. [CrossRef] 
23. Nunes-Pereira, J.; Sencadas, V.; Correia, V.; Cardoso, V.F.; Han, W.; Rocha, J.G.; Lanceros-Mendez, S. Energy harvesting performance of BaTiO3/poly(vinylidene fluoride-trifluoroethylene) spin coated nanocomposites. Compos. Part B Eng. 2015, 72, 130-136. [CrossRef]

24. Ni, J.; Zhao, Y.; Li, L.; Mai, L. Ultrathin $\mathrm{MoO}_{2}$ nanosheets for superior lithium storage. Nano Energy 2015, 11, 129-135. [CrossRef]

25. Ponnamma, D.; Al-Maadeed, M.A.A. Influence of $\mathrm{BaTiO}_{3} /$ white graphene filler synergy on the energy harvesting performance of a piezoelectric polymer nanocomposite. Sustain. Energy Fuels 2019, 3, 774-785. [CrossRef]

26. Sabry, R.S.; Hussein, A.D. PVDF: $\mathrm{ZnO} / \mathrm{BaTiO}_{3}$ as high out-put piezoelectric nanogenerator. Polym. Test. $2019,79,106001$. [CrossRef]

27. Bijelić, J.; Stanković, A.; Medvidović-Kosanović, M.; Marković, B.; Cop, P.; Sun, Y.; Hajra, S.; Sahu, M.; Vukmirović, J.; Marković, D.; et al. Rational sol-gel-based synthesis design and magnetic, dielectric, and optical properties study of nanocrystalline $\mathrm{Sr}_{3} \mathrm{CO}_{2} \mathrm{WO}_{9}$ triple perovskite. J. Phys. Chem. C 2020, 124, 12794-12807. [CrossRef]

28. Harwood, M.G.; Popper, P.; Rushman, D.F. Curie point of barium titanate. Nature 1947, 160, 58-59. [CrossRef]

29. Matoba, R.; Hayashi, Y.; Takizawa, H. Preparation of barium titanate powders by microwave-assisted liquid phase process at ambient pressure. J. Ceram. Soc. Jpn. 2009, 117, 388-391. [CrossRef]

30. Hajra, S.; Sahoo, S.; Das, R.; Choudhary, R. Structural, dielectric and impedance characteristics of $\left(\mathrm{Bi}_{0.5} \mathrm{Na}_{0.5}\right) \mathrm{TiO}_{3}-\mathrm{BaTiO}_{3}$ electronic system. J. Alloys Compd. 2018, 750, 507-514. [CrossRef]

31. Hajra, S.; Sahoo, S.; Choudhary, R.N.P. Fabrication and electrical characterization of $\left(\mathrm{Bi}_{0.49} \mathrm{Na}_{0.49} \mathrm{Ba}_{0.02}\right) \mathrm{TiO}_{3}-\mathrm{PVDF}$ thin film composites. J. Polym. Res. 2019, 26, 14. [CrossRef]

32. Dash, S.; Choudhary, R.N.P.; Kumar, A.; Goswami, M.N. Enhanced dielectric properties and theoretical modeling of PVDFceramic composites. J. Mater. Sci. Mater. Electron. 2019, 30, 19309-19318. [CrossRef]

33. Kuo, D.-H.; Chang, C.-C.; Su, T.-Y.; Wang, W.-K.; Lin, B.-Y. Dielectric properties of three ceramic/epoxy composites. Mater. Chem. Phys. 2004, 85, 201-206. [CrossRef] 